\title{
Rigorous Solutions of Electromagnetic Problems Involving Hundreds of Millions of Unknowns
}

\author{
Özgür Ergül' and Levent Gürel ${ }^{2,3}$ \\ 1 Department of Mathematics and Statistics \\ University of Strathclyde, Glasgow, UK \\ ${ }^{2}$ Department of Electrical and Electronics Engineering \\ ${ }^{3}$ Computational Electromagnetics Research Center (BiLCEM) \\ Bilkent University, TR-06800, Bilkent, Ankara, Turkey \\ E-mail: ozgur.ergul@strath.ac.uk, Igurel@ee.bilkent.edu.tr
}

\begin{abstract}
Accurate simulations of real-life electromagnetic problems with integral equations require the solution of dense matrix equations involving millions of unknowns. Solutions of these extremely large problems cannot be easily achieved, even when using the most powerful computers with state-of-the-art technology. Hence, many electromagnetic problems in the literature have been solved by resorting to various approximation techniques, without controllable error. In this paper, we present fullwave solutions of scattering problems discretized with hundreds of millions of unknowns by employing a parallel implementation of the Multilevel Fast Multipole Algorithm. Various examples involving canonical and complicated objects, including scatterers larger than $1000 \lambda$, are presented, in order to demonstrate the feasibility of accurately solving large-scale problems on relatively inexpensive computing platforms.
\end{abstract}

Keywords: Electromagnetic fields; electromagnetic scattering; integral equations; iterative methods; parallel algorithms; multilevel fast multipole algorithm

\section{Introduction}

$\mathbf{E}$ lectromagnetic scattering and radiation problems can be rigorously formulated with the integral-equation forms of Maxwell's equations, which describe the relationships between electromagnetic fields and sources. Since analytical solutions are possible only for a few objects (such as a sphere [1]), numerical techniques, with the help of computers, are required to solve electromagnetic problems. Discretizations of objects lead to dense matrix equations, which can be interpreted as mathematical representations of electromagnetic interactions. Solutions of matrix equations provide discretized versions of unknown current distributions, from which electric and magnetic fields can be computed everywhere.

An accurate solution of an electromagnetic problem requires the discretization of the object with elements small compared to the wavelength. Real-life problems often involve large objects, with dimensions of hundreds of wavelengths, and they lead to discretizations with millions of unknowns. Since conventional direct methods are insufficient to solve those large-scale problems, various acceleration methods, such as the Fast Multipole Method (FMM) [2] and the Multilevel Fast Multipole Algorithm (MLFMA) [3, 4], have been proposed in the literature. Employing FMM and MLFMA on parallel computers has enabled the solution of many real-life problems [5-13], such as scattering from ordinary and radar-eluding stealth airborne targets, radiation from antennas and electronic devices, and transmission through frequency-selective metamaterials, photonic crystals, and optical imaging systems, to name a few. Nevertheless, due to their large sizes, many other electromagnetic problems cannot be solved even with acceleration methods, and only approximate solutions of those problems are available in the literature.

Recently, we showed that an efficient parallelization of MLFMA can provide full-wave solutions of extremely large electromagnetic problems, involving more than 370 million unknowns, on relatively inexpensive computing platforms. Figure 1 depicts the sizes of dense matrix equations solved since 1999 using parallel implementations of the MLFMA. The number of unknowns is also plotted in detail as a function of time, from January 2007 to December 2009. In 2007, optimization of a hybrid parallelization technique [7] made it possible to solve very large problems involving 33 to 53 million unknowns. Development of a hierarchical parallelization technique in the beginning of 2008 [10] enabled the solution of even larger problems, involving as many as 85 million unknowns. With the optimization of the hierarchical parallelization technique, the number of unknowns was then successfully increased to more than 200 million before the end of 2008 [12], and to more than 370 million before the end of 2009 . 


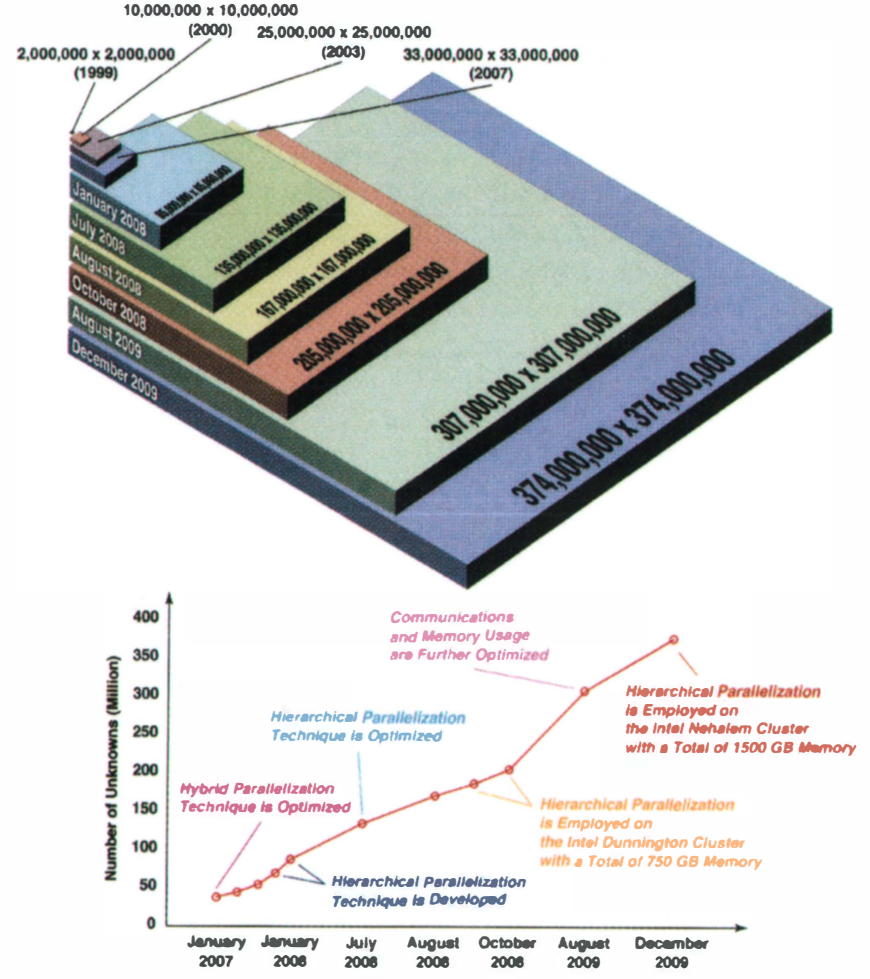

Figure 1. Solutions of large matrix equations via parallel implementations of MLFMA since 1999. The number of unknowns is plotted as a function of time. By December 2009, the hierarchical parallelization technique enabled the solution of extremely large electromagnetic problems involving more than 370 million unknowns on relatively inexpensive computing platforms.

In this paper, we present rigorous and accurate solutions of extremely large scattering problems involving three-dimensional canonical and complicated metallic objects. By employing a scalable parallel implementation of the MLFMA using the hierarchical partitioning strategy, we are able to solve both efficiently and accurately electromagnetic problems discretized with hundreds of millions of unknowns. We present many examples to demonstrate the feasibility of accurately solving large-scale problems on relatively inexpensive computing platforms, without resorting to approximation techniques. Since they were obtained by using a full-wave solver, numerical results presented in this paper can be used for benchmarking purposes, i.e., for measuring the accuracy of novel solvers in the area of computational electromagnetics.

\section{Fast and Accurate Simulation Environment Based on Parallel MLFMA}

The MLFMA and its efficient parallelization using the hierarchical partitioning strategy were extensively discussed in [4] and [12]. In this section, we summarize only the major components of our simulation environment, as well as computational requirements and error sources, in the context of developing an efficient and accurate electromagnetic solver.

\subsection{Integral-Equation Formulations}

Integral equations are derived directly from Maxwell's equations, without any approximation. For a perfectly conducting object with surface $S$, the electric-field integral equation (EFIE) and the magnetic-field integral equation (MFIE) are derived as [14]

$$
-\hat{\mathbf{n}} \times \hat{\mathbf{n}} \times i k \int_{S} d \mathbf{r}^{\prime}\left[\mathbf{J}\left(\mathbf{r}^{\prime}\right)+\frac{1}{k^{2}} \nabla^{\prime} \cdot \mathbf{J}\left(\mathbf{r}^{\prime}\right) \nabla\right] g\left(\mathbf{r}, \mathbf{r}^{\prime}\right)=\hat{\mathbf{n}} \times \hat{\mathbf{n}} \times \eta^{-1} \mathbf{E}^{i n c}(\mathbf{r})
$$

and

$$
-\frac{\Omega_{o}}{4 \pi} \mathbf{J}(\mathbf{r})+\hat{\mathbf{n}} \times \int_{S, P V} d \mathbf{r}^{\prime} \mathbf{J}\left(\mathbf{r}^{\prime}\right) \times \nabla^{\prime} g\left(\mathbf{r}, \mathbf{r}^{\prime}\right)=-\hat{\mathbf{n}} \times \mathbf{H}^{i n c}(\mathbf{r})
$$

respectively, in phasor notation, using the $e^{-i \omega t}$ convention. In Equations (1) and (2), $\mathbf{J}(\mathbf{r})=\hat{\mathbf{n}} \times \mathbf{H}(\mathbf{r})$ is the electric current induced on the surface $S, \hat{\mathbf{n}}$ is the normal unit vector, $\Omega_{o}$ is the external solid angle, $k=2 \pi / \lambda=\omega \sqrt{\varepsilon \mu}$ is the wavenumber, $\eta=\sqrt{\mu / \varepsilon}$ is the intrinsic impedance, $\mathbf{E}^{i n c}(\mathbf{r})$ and $\mathbf{H}^{i n c}(\mathbf{r})$ are incident electric and magnetic fields created by external sources, and

$$
g\left(\mathbf{r}, \mathbf{r}^{\prime}\right)=\frac{\exp \left(i k\left|\mathbf{r}-\mathbf{r}^{\prime}\right|\right)}{4 \pi\left|\mathbf{r}-\mathbf{r}^{\prime}\right|}
$$

is the scalar Green's function.

In this paper, we consider closed conducting surfaces, which can be formulated with the combined-field integral equation (CFIE). The combined-field integral equation is a convex combination of the electric-field integral equation and the magnetic-field integral equation [15], i.e., $\mathrm{CFIE}=\alpha \mathrm{EFIE}+(1-\alpha) \mathrm{MFIE}$, where $\alpha$ is a parameter between 0 and 1 . We chose $\alpha=0.2$, which usually leads to the most efficient iterative solutions [16]. In addition to its well-conditioned nature [4], the combined-field integral equation is free of the internal resonances experienced by the electric-field integral equation and the magnetic-field integral equation [15].

\subsection{Matrix Equations and Iterative Solutions}

Discretizations of integral equations lead to $N \times N$ dense matrix equations in the form of

$$
\overline{\mathbf{Z}} \cdot \mathbf{a}=\mathbf{v},
$$

where elements of the impedance matrix $\overline{\mathbf{Z}}$ can be interpreted as electromagnetic interactions between discretization elements. The right-hand-side vector $\mathbf{v}$ is obtained by testing incident fields due to external sources. In order to compute expansion coefficients $\mathrm{a}[n]$ for $n=1,2, \ldots, N$, the matrix equation in Equation (4) can be solved by using an iterative algorithm, where the solution is expanded in a Krylov subspace, i.e.,

$$
\mathbf{a} \in \operatorname{Span}\left\{\mathbf{v}, \overline{\mathbf{Z}} \cdot \mathbf{v}, \overline{\mathbf{Z}}^{2} \cdot \mathbf{v}, \ldots, \overline{\mathbf{Z}}^{j} \cdot \mathbf{v}\right\}
$$

To reduce the number of iterations $j$, it is common to employ various preconditioning techniques [17] and to transform the original matrix equation into a better-conditioned equation, such as 


$$
\overline{\mathbf{P}}^{-1} \cdot \overline{\mathbf{Z}} \cdot \mathbf{a}=\overline{\mathbf{P}}^{-1} \cdot \mathbf{v}
$$

In Equation (6), $\overline{\mathbf{P}} \approx \overline{\mathbf{Z}}$ is a preconditioner matrix, which is obtained by a partial or approximate factorization of $\overline{\mathbf{Z}}$.

For the discretization, we use small planar triangles on which Rao-Wilton-Glisson (RWG) functions [18] are defined to expand equivalent currents and to test boundary conditions. According to our experience, the size of the triangles should not exceed $\lambda / 10$ if $1 \%$ overall error is to be achieved. More-accurate simulations require discretizations with smaller triangles or the use of higherorder basis functions, such as linear-linear functions [16, 19]. Among various iterative methods, we prefer the bi-conjugate-gradient-stabilized (BiCGStab) algorithm [20], which is known to provide rapid solutions for the combined-field integral equation [21]. Iterative solutions are further accelerated by employing block-diagonal preconditioners that are constructed from the self interactions of the lowest-level subdomains in MLFMA [3].

\subsection{Multilevel Fast Multipole Algorithm}

Iterative solutions of matrix equations require matrix-vector multiplications, i.e., $\mathbf{y}=\overline{\mathbf{Z}} \cdot \mathbf{x}$, which can be performed directly with $\mathcal{O}\left(N^{2}\right)$ complexity. For large-scale problems, direct multiplications render the solution impossible with limited computational resources. In this study, we use the MLFMA $[3,4]$ to perform matrix-vector multiplications in $\mathcal{O}(N \log N)$ time using $\mathcal{O}(N \log N)$ memory, without deterioration in the accuracy of results. Considering the fact that matrix elements can be interpreted as electromagnetic interactions between pairs of discretization elements, i.e., basis and testing functions, the MLFMA performs far-field interactions in a group-by-group manner using the factorization and diagonalization of the Green's function [2].

In the MLFMA, a tree structure of $L=\mathcal{O}(\log N)$ levels is constructed by recursively dividing the computational domain into subdomains. Matrix-vector multiplications are then decomposed into two parts as

$$
\mathbf{y}=\overline{\mathbf{Z}}_{N F} \cdot \mathbf{x}+\overline{\mathbf{Z}}_{F F} \cdot \mathbf{x},
$$

where only the near-field interactions $\left(\overline{\mathbf{Z}}_{N F} \cdot \mathbf{x}\right)$ between close subdomains are performed directly. The far-field interactions $\left(\overline{\mathbf{Z}}_{F F} \cdot \mathbf{x}\right)$ between distant subdomains are calculated efficiently in three stages, called aggregation, translation, and disaggregation. In the aggregation stage, radiated fields of subdomains are calculated from the bottom of the tree structure to the highest level. Next, in the translation stage, radiated fields are translated into incoming fields via diagonal translation operators. Finally, in the disaggregation stage, total incoming fields are calculated from the top of the tree structure to the lowest level. At the lowest level, incoming fields are received by testing functions to complete the matrixvector multiplication.

\subsection{On the Error Sources}

In addition to simultaneous discretizations of surfaces and integral equations, solutions via the MLFMA involve various error sources, which must be carefully controlled to ensure that reliable simulations are performed. In our implementations, near-field interactions are calculated by using singularity extraction techniques [22-25] and adaptive integration methods [26] to satisfy preset error bounds. For far-field interactions, we use the excessbandwidth formula [27] to determine truncation numbers and sampling rates for radiated and incoming fields. We also use various techniques in order to suppress additional errors due to interpolation and anterpolation operations [28, 29]. Finally, the targeted residual error for iterative convergence is selected carefully in accordance with the errors in near-field and far-field interactions.

\subsection{On the Computational Requirements}

The solution part of the MLFMA involving multiple iterations usually dominates the setup part in terms of the processing time. The calculation of radiation and receiving patterns of basis and testing functions, translation operators, and the right-hand-side vector in the setup part usually requires negligible time. Nevertheless, the setup time is also significant, due to the calculation of near-field interactions. A majority of memory in the MLFMA is used for the radiation and receiving patterns of basis and testing functions, near-field interactions, and aggregation/disaggregation arrays required during matrix-vector multiplications. We note that as opposed to most other implementations of the MLFMA, we calculate and store the radiation and receiving patterns of basis and testing functions during the setup of the program, and we use them efficiently during iterations. Calculating patterns on the fly in each matrix-vector multiplication without storing them would decrease the memory requirements, but the processing time would significantly increase.

\subsection{Parallelization}

By reducing the complexity of matrix-vector multiplications from $\mathcal{O}\left(N^{2}\right)$ to $\mathcal{O}(N \log N)$, the MLFMA enables the solution of large problems involving hundreds of thousands to a few millions of unknowns. Nevertheless, accurate solutions of real-life problems often require discretizations with tens and even hundreds of millions of unknowns. In order to handle such extremely large problems, the MLFMA must be parallelized on a cluster of computers. Unfortunately, parallelization of the MLFMA is not trivial, as opposed to some other algorithms with higher complexities, such as the FMM, which has been parallelized very successfully [13]. Specifically, distributing the multilevel tree structure among processors has been a major bottleneck for the efficient parallelization of the MLFMA.

In a typical tree structure of the MLFMA, the lowest level involves $\mathcal{O}(N)$ subdomains with dimensions of the order of a wavelength, and the number of subdomains decreases by a factor of four from one level to the next-higher level. On the other hand, the number of samples required for the factorization and diagonalization of the Green's function depends on the size of the subdomains as measured by the wavelength. Consequently, the number of samples increases by a factor of four from one level to the nexthigher level. This way, the number of subdomains and the number of samples balance each other, and all levels in the MLFMA have an equal workload, with $\mathcal{O}(N)$ complexity. An efficient parallelization of the MLFMA should therefore consider the best possible partitioning at each level. 
In a simple parallelization of the MLFMA, subdomains are distributed among processors at all levels. Such a technique provides efficient parallelization for small numbers of processors, usually fewer than 16 . However, the efficiency drops drastically for more processors, due to poor load balancing. This is because small numbers of subdomains in the higher levels of the MLFMA cannot be distributed evenly among processors. In addition, dense communications are required among processors. We note that low parallelization efficiency not only increases the processing time, but also restricts the size of problems that can be solved. As a remedy, a hybrid technique was proposed to improve the parallelization of the MLFMA [6, 10]. This technique is based on using an appropriate partitioning scheme for the higher levels of the MLFMA by distributing samples instead of subdomains. Due to the improved load balancing in the higher levels, the hybrid technique significantly increases the parallelization efficiency compared to the simple technique. Nevertheless, the hybrid technique also fails to provide efficient solutions when the number of processors is larger than 32 [8].

Recently, we proposed a hierarchical parallelization technique that is based on improved partitioning at each level of the MLFMA [8, 12]. In this technique, both subdomains and their samples are partitioned among processors by employing load-balancing algorithms. Although changing the partitioning between levels bears an additional cost, the hierarchical technique offers a higher parallelization efficiency than previous approaches. Specifically, the hierarchical partitioning strategy provides two important advantages compared to previous parallelization techniques for the MLFMA. First, partitioning both subdomains and samples of fields leads to improved load balancing among processors at each level. Second, communications between processors are reduced, i.e., the average package size is enlarged, the number of communication events is reduced, and the communication time is significantly shortened. Details of the hierarchical partitioning strategy and comparisons with previous parallelization techniques can be found in [12].

\subsection{Computing Platforms}

Solutions presented in this paper were performed on three relatively inexpensive computing platforms with distributed-memory architectures. The Harpertown cluster at Bilkent University Computational Electromagnetics Research Center (BiLCEM), Ankara, Turkey, consists of 16 computing nodes, and each node has $32 \mathrm{~GB}$ of memory and two Intel Xeon Harpertown quad-core processors with $3.00 \mathrm{GHz}$ clock rates. The Dunnington cluster in Swindon, UK, also consists of 16 computing nodes, but each node has $48 \mathrm{~GB}$ of memory and two Intel Xeon Dunnington hexa-core processors with $2.40 \mathrm{GHz}$ clock rates. Finally, the Nehalem cluster at the Turkish Academic Network and Information Center (ULAKBIM), Ankara, Turkey, consists of 64 computing nodes, and each node has $24 \mathrm{~GB}$ of memory and two Intel Xeon Nehalem quad-core processors with $2.67 \mathrm{GHz}$ clock rates. For all solutions on the Harpertown and Dunnington clusters, we employed four cores per node (a total of 64 cores), while we employed only one core per node on the Nehalem cluster (again, a total of 64 cores).

\section{Results}

In this section, we present the solution of large-scale scattering problems involving various canonical and complicated objects. In all examples, the targets were located in free space.

\subsection{Sphere}

In order to demonstrate the accuracy of the developed simulation environment, Figure 2 presents the solution of a scattering problem involving a metallic sphere of diameter $a_{s}=60 \mathrm{~cm}$. The sphere was illuminated by a plane wave propagating in the $-x$ direction at $280 \mathrm{GHz}$. The diameter of the sphere corresponded to $560 \lambda$ at this frequency. Discretization of the sphere using the RWG functions on $\lambda / 10$ triangles led to a $374,490,624 \times 374,490,624$ dense matrix equation. Both nearfield and far-field interactions were calculated with a maximum of $1 \%$ error. Convergence to a residual error of 0.001 was achieved in 31 iterations. For the solution, which took about 19 hours, an 11level MLFMA was parallelized on the Nehalem cluster. Figure 2 presents the normalized bistatic radar cross section ( $\left.\mathrm{RCS} / a_{s}^{2}\right)$ in decibels $(\mathrm{dB})$ on the $x-y$ plane as a function of the bistatic angle $\phi$ from $0^{\circ}$ to $360^{\circ}$, where $0^{\circ}$ and $180^{\circ}$ corresponded to the backscattering and forward-scattering directions, respectively. In the inset of Figure 2, computational values provided by the parallel MLFMA implementation were compared with an analytical Mieseries solution [1] from $179^{\circ}$ to $180^{\circ}$, where we observed perfect agreement. We also note that a direct solution of a similar problem involving 375 million unknowns without using the MLFMA would require 1.1 exabytes ( 1 exabyte $\left.=10^{9} \mathrm{~GB}\right)$ of memory, and it would take more than 2000 years just to construct the matrix equation on the same Nehalem cluster. In addition, such a hypothetical solution would not be able to attain $1 \%$ error, due to accumulation of rounding errors in finite-precision arithmetic.

\subsection{Other Canonical and Complicated Objects}

In addition to the sphere, we present the solutions of scattering problems involving the four metallic objects depicted in Fig-

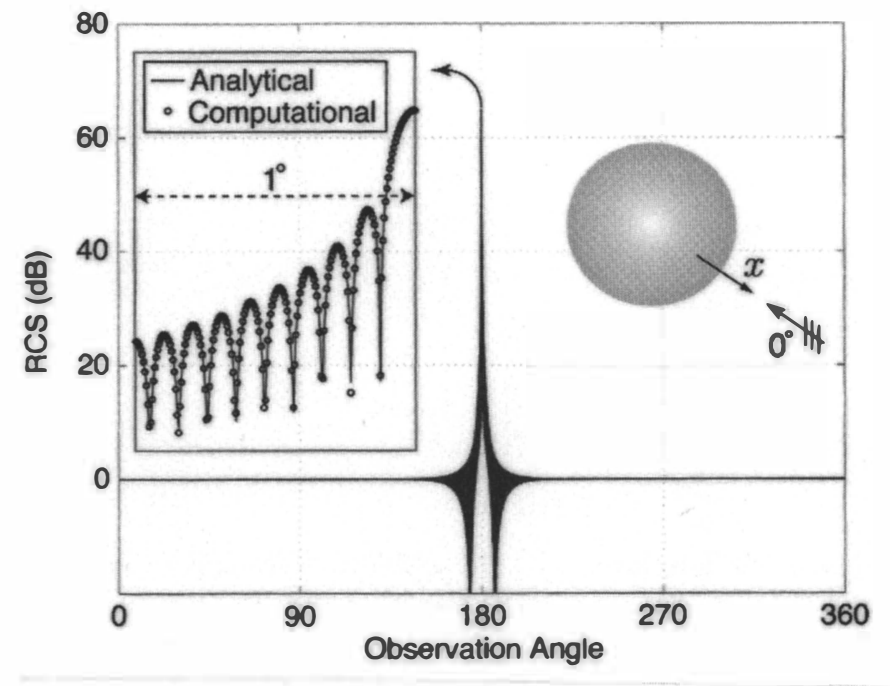

Figure 2. The solution of a scattering problem involving a metallic sphere of diameter $a_{s}=60 \mathrm{~cm}$ at $280 \mathrm{GHz}$. The normalized RCS (dB) is plotted as a function of the bistatic angle from $0^{\circ}$ to $360^{\circ}$, where $180^{\circ}$ corresponds to the forwardscattering direction. Computational values provided by the parallel MLFMA implementation with maximum $1 \%$ error agreed well with an analytical Mie-series solution from $179^{\circ}$ to $180^{\circ}$ (inset). 


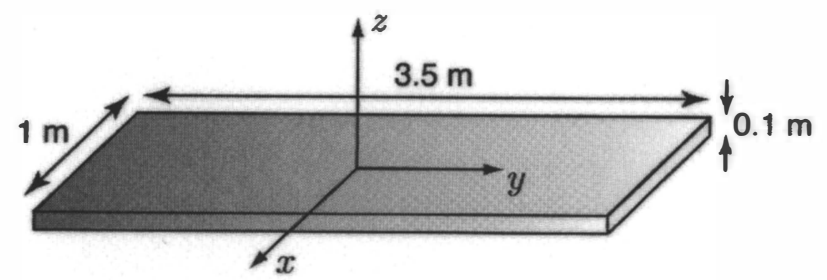

Figure 3a. A large metallic rectangular box, the scattering problem for which was solved with the parallel MLFMA implementation.
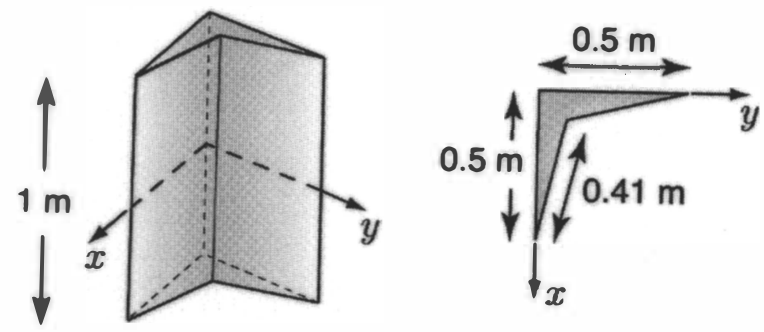

Figure 3b. A large metallic wing-shaped object, the scattering problem for which was solved with the parallel MLFMA implementation.

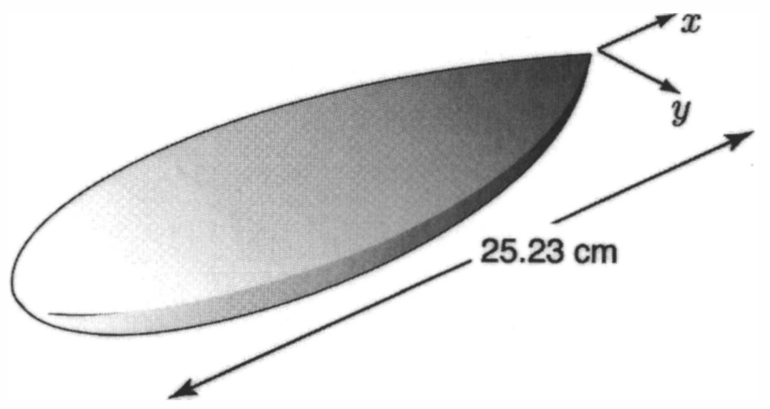

Figure 3c. The large metallic NASA almond, the scattering problem for which was solved with the parallel MLFMA implementation.

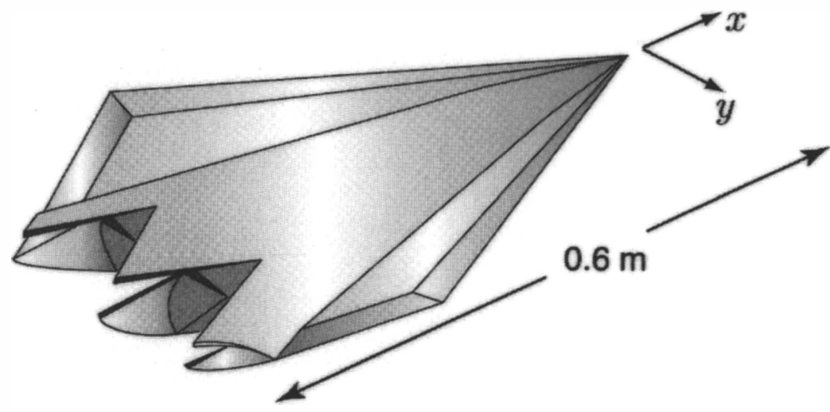

Figure 3d. The large metallic Flamme object, the scattering problem for which was solved with the parallel MLFMA implementation.

ure 3, namely, a rectangular box, a wing-shaped object with sharp edges and corners, the NASA Almond [30], and the stealth airborne target Flamme [31]. The problems were discretized with the RWG functions on $\lambda / 10$ triangles. For all solutions, near-field and far-field interactions were calculated with a maximum of $1 \%$ error, and the target residual error for the iterative convergence was set to 0.001 .

\subsubsection{Rectangular Box}

The rectangular box was investigated at $75 \mathrm{GHz}$. At this frequency, the size of the box corresponded to $875 \lambda$, and its discretization led to matrix equations involving $174,489,600$ unknowns. The box was illuminated by two plane waves propagating on the $x-z$ plane at $30^{\circ}$ and $60^{\circ}$ angles from the $z$ axis, with the electric field polarized in the $\phi$ direction. The total number of iterations was 32 , and two solutions were performed in a total of 10 hours on the Dunnington cluster. Figure 4 depicts the co-polar bistatic RCS ( $\mathrm{dBms}$ stands for $\mathrm{dBm}^{2}$ ) on the $x-z$ plane as a function of the bistatic angle $\theta$. In addition to the forward-scattering direction, the RCS values made peaks at two specular reflection directions, i.e., at $150^{\circ}$ and $330^{\circ}$ for the $30^{\circ}$ illumination, and at $120^{\circ}$ and $300^{\circ}$ for the $60^{\circ}$ illumination. Both the directions and the magnitudes of these peaks were consistent with reflections from the large top surface and the small side surface.

\subsubsection{Wing-Shaped Object}

The wing-shaped object with a maximum dimension of $1 \mathrm{~m}$ was investigated at $150 \mathrm{GHz}$. The object was discretized with

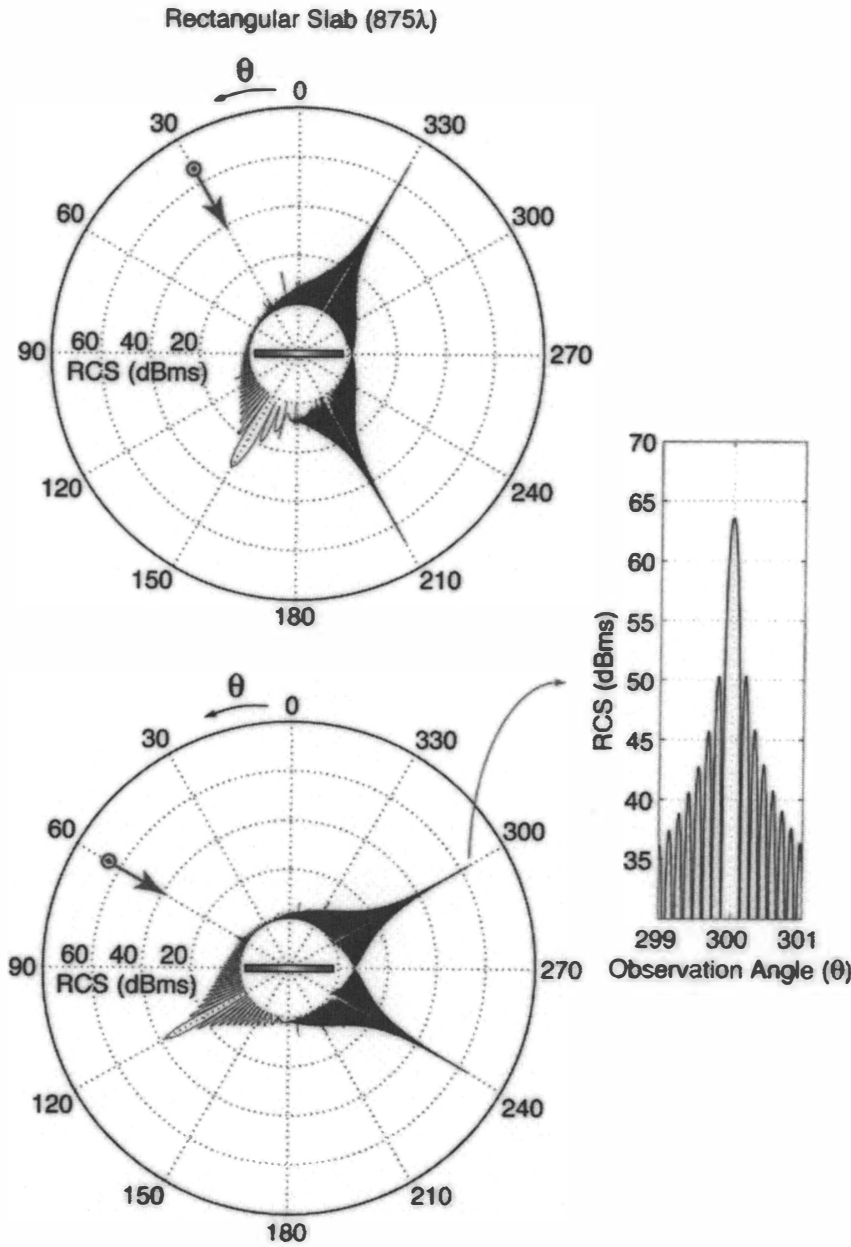

Figure 4. The co-polar bistatic RCS (dBms) of the rectangular box in Figure 3a at $75 \mathrm{GHz}$ on the $x-z$ plane. The box was illuminated by plane waves propagating on the $x-z$ plane at $30^{\circ}$ and $60^{\circ}$ angles from the $z$ axis, with the electric field polarized in the $\phi$ direction. 


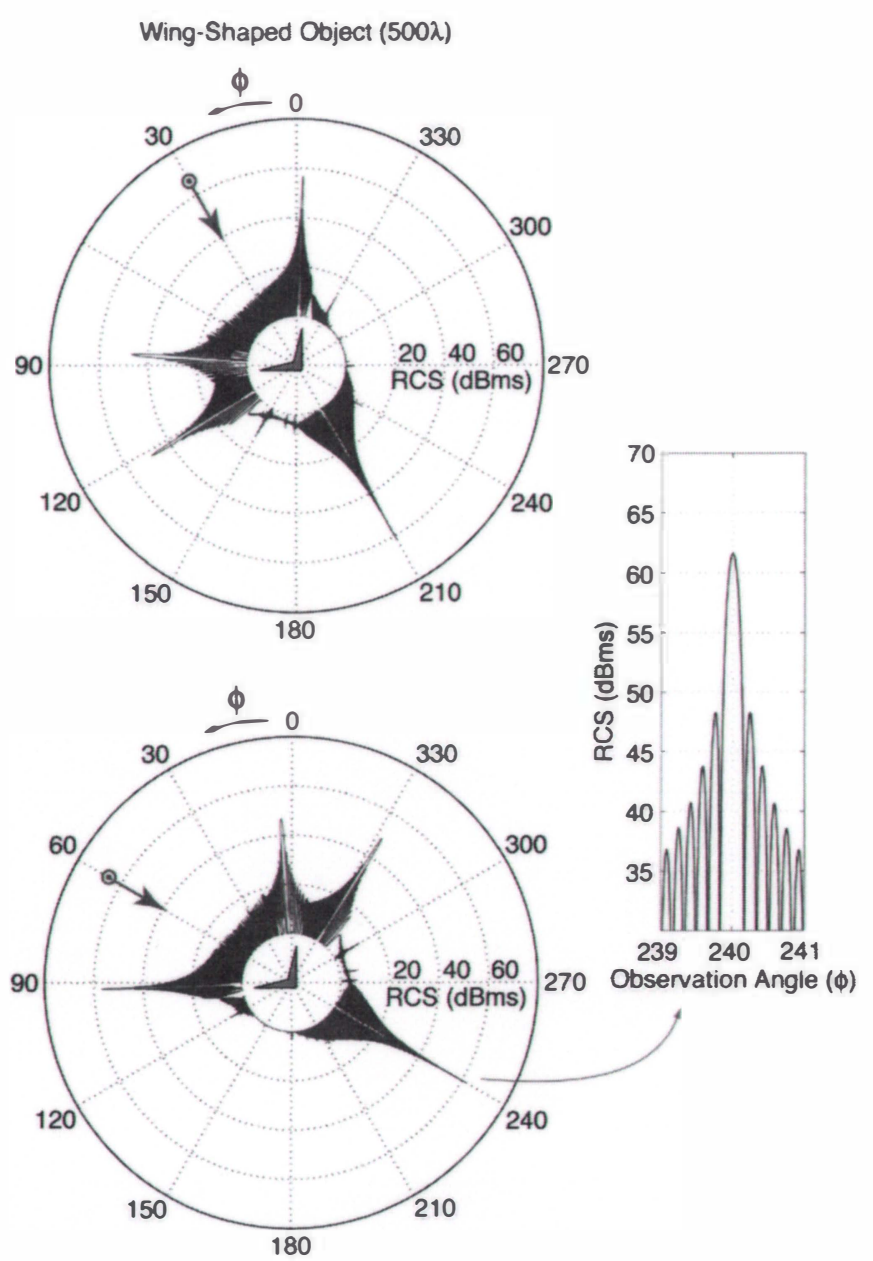

Figure 5. The co-polar bistatic RCS (dBms) of the wing-shaped object in Figure 3b at $150 \mathrm{GHz}$ on the $x-y$ plane. The object was illuminated by plane waves propagating on the $x-y$ plane at $30^{\circ}$ and $60^{\circ}$ angles from the $x$ axis, with the electric field polarized in the $\theta$ direction.

$172,228,608$ unknowns, and it was illuminated by two plane waves propagating on the $x-y$ plane at $30^{\circ}$ and $60^{\circ}$ angles from the $x$ axis. The electric field was polarized in the $\theta$ direction. The total number of iterations was 36 , and two solutions were performed in a total of 12 hours on the Dunnington cluster. Figure 5 depicts the co-polar bistatic RCS (dBms) on the $x-y$ plane as a function of the bistatic angle $\phi$. We observed that the RCS values of the wingshaped object made peaks in three directions due to specular reflections, in addition to the forward-scattering direction. Two of these peaks were due to specular reflections from the two large flat surfaces of the wing, but the third peak interestingly corresponded to double reflection from both surfaces. This result can be used to benchmark single-bounce and multi-bounce high-frequency approximations.

\subsubsection{NASA Almond and the Flamme Discretized with More than 100 Million Unknowns}

Next, we present the solution of scattering problems involving two important metallic targets from the literature, namely, the
NASA Almond [30] and the stealth airborne target Flamme [31]. The maximum dimension of the NASA Almond was $25.23 \mathrm{~cm}$ and this corresponded to $715 \lambda$ at $850 \mathrm{GHz}$. The scaled size of the Flamme was $0.6 \mathrm{~m}$, and this corresponded to $720 \lambda$ at $360 \mathrm{GHz}$. Discretizations of the NASA Almond and the Flamme with $\lambda / 10$ mesh size led to matrix equations involving $125,167,104$ and $134,741,760$ unknowns, respectively. Both targets were located on the $x-y$ plane, such that their noses were directed towards the $x$ axis, and they were illuminated by various plane waves propagating on the $x-y$ plane. We considered both $\phi$ and $\theta$ polarizations of the incident electric field. Figure 6 presents the number of BiCGStab iterations and the total processing time (including setup and iterative solution parts) in seconds when solutions were performed using an 11-level MLFMA on the Harpertown cluster. The number of iterations and the processing time are plotted as functions of the illumination angle measured from the $x$ axis. For the Flamme, the number of iterations increased with the increasing illumination angle, due to the resonant characteristics of the cavity at the back of the target. This was not observed for the NASA Almond, which had a convex surface with a more regular shape.
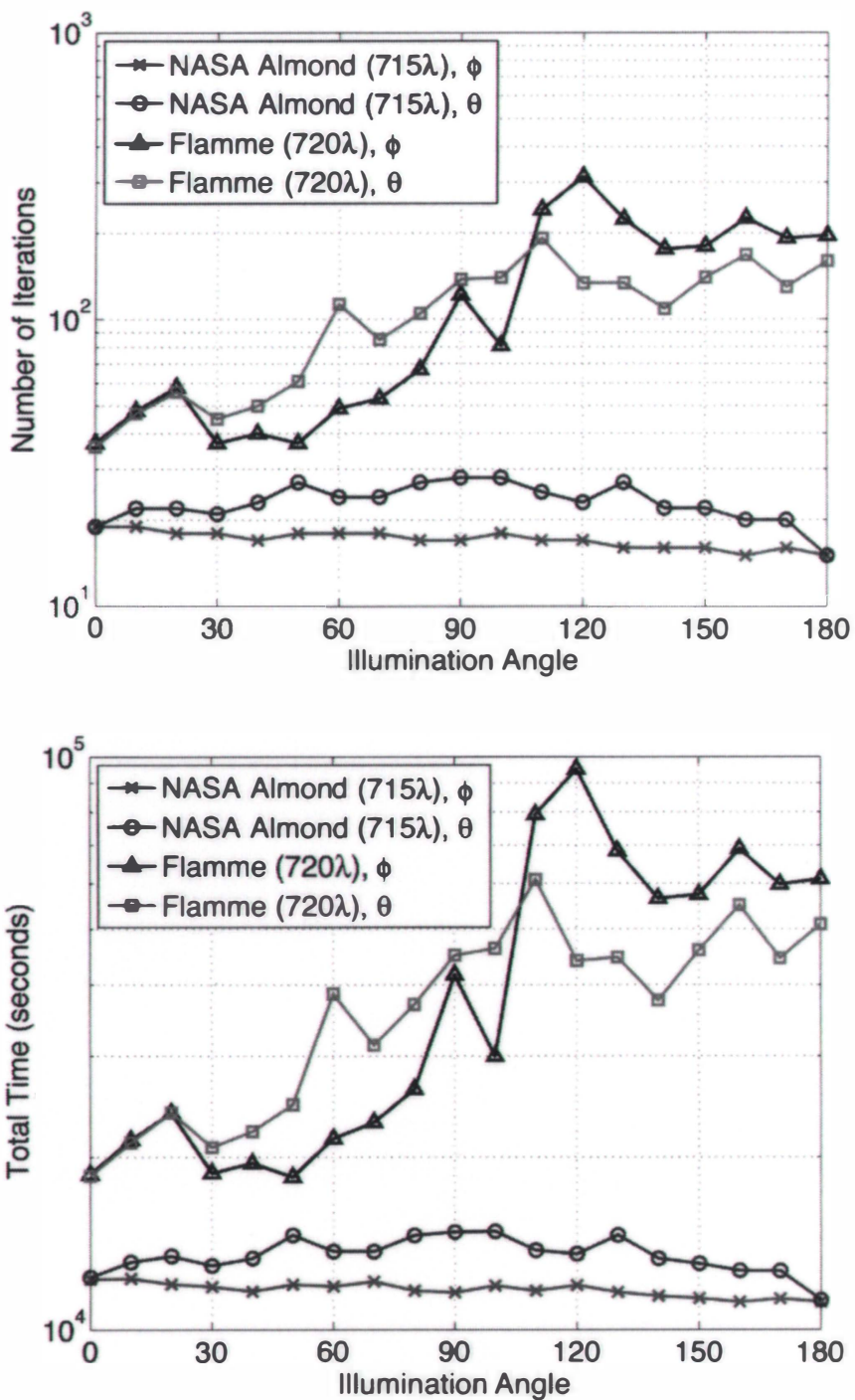

Figure 6. Solutions of scattering problems involving the NASA Almond (Figure 3c) at $850 \mathrm{GHz}$, and the Flamme (Figure 3d) at $360 \mathrm{GHz}$. Both targets were discretized with more than 100 million unknowns. (a) The number of BiCGStab iterations and (b) the total processing time were plotted with respect to the illumination angle measured from the $x$ axis. Both $\phi$ and $\theta$ polarizations of the incident electric field were considered. 


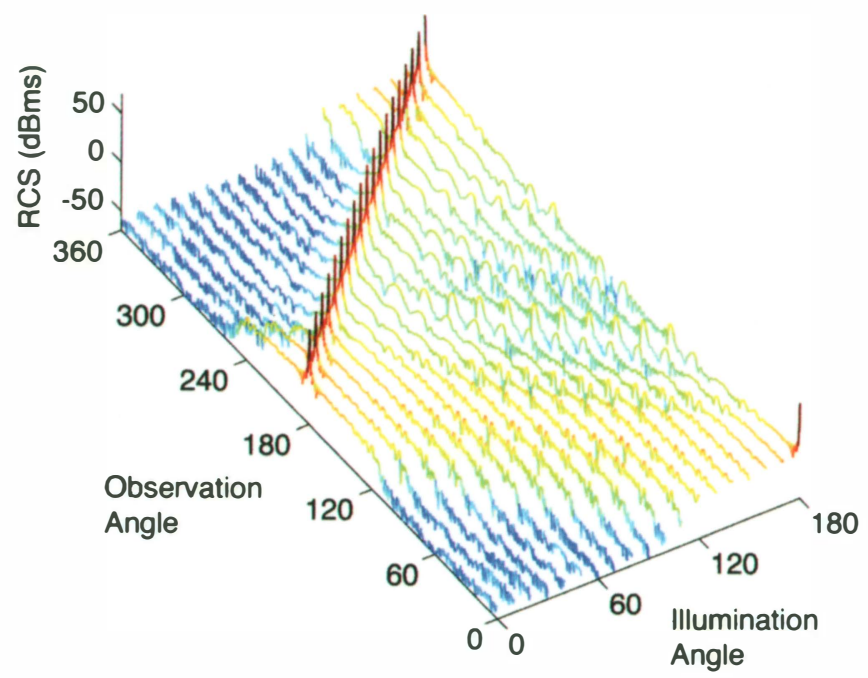

NASA Almond $(\theta \theta, 715 \lambda)$

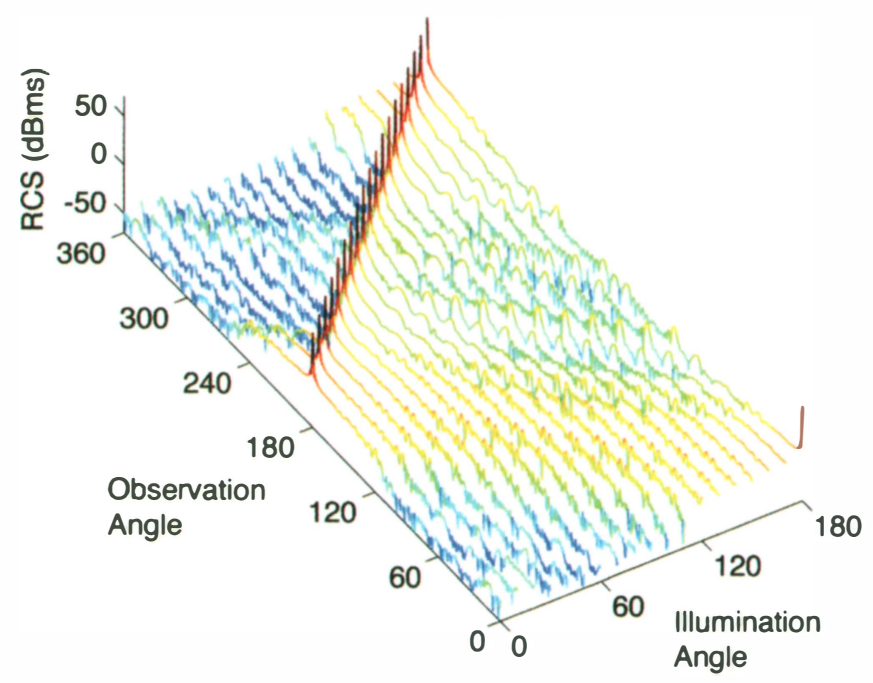

Figure 7. The co-polar bistatic RCS (dBms) of the NASA Almond in Figure 3c at $850 \mathrm{GHz}$. The RCS was plotted on the $x-y$ plane as a function of the observation and illumination angles when the incident electric field was polarized in (a) the $\phi$ and (b) the $\theta$ directions.

Figures 7 and 8 present the co-polar bistatic RCS (dBms) of the NASA Almond and the Flamme, respectively, on the $x-y$ plane as a function of observation and illumination angles. Figure 7 shows that in addition to the forward-scattering direction, the RCS of the NASA Almond was quite high in a range of observation angles, depending on the illumination angle. For both $\phi$ and $\theta$ polarizations, we observed significantly large RCS values from $\left(120^{\circ}-\phi_{i}\right)$ to $\left(240^{\circ}-\phi_{i}\right)$, where $\phi_{i}$ is the illumination angle. As depicted in Figure 8, the bistatic RCS of the Flamme was very different from the bistatic RCS of the NASA Almond. Specifically, the Flamme RCS exhibited several peaks in various directions, depending on the illumination.

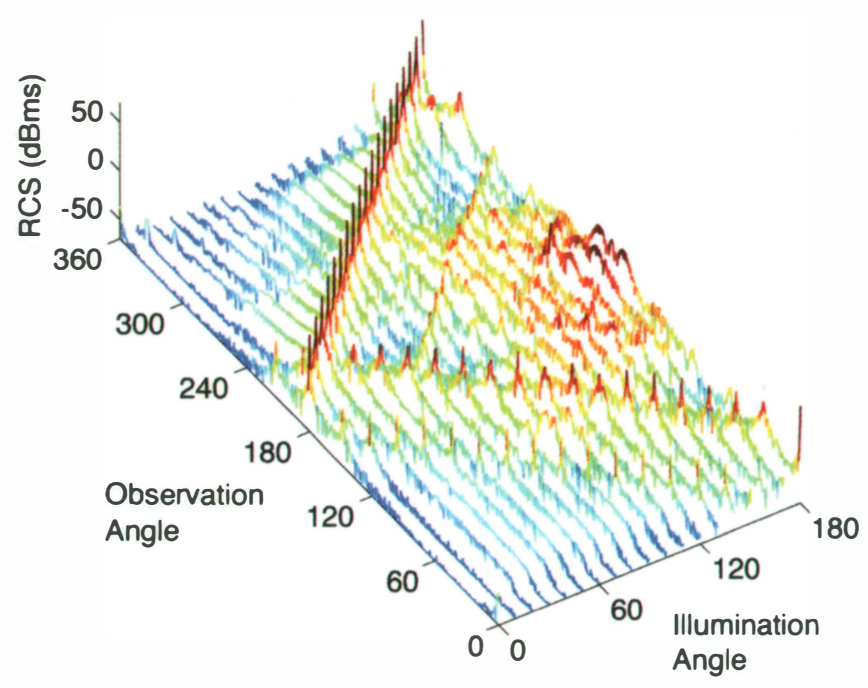

Flamme $(\theta \theta, 720 \lambda)$

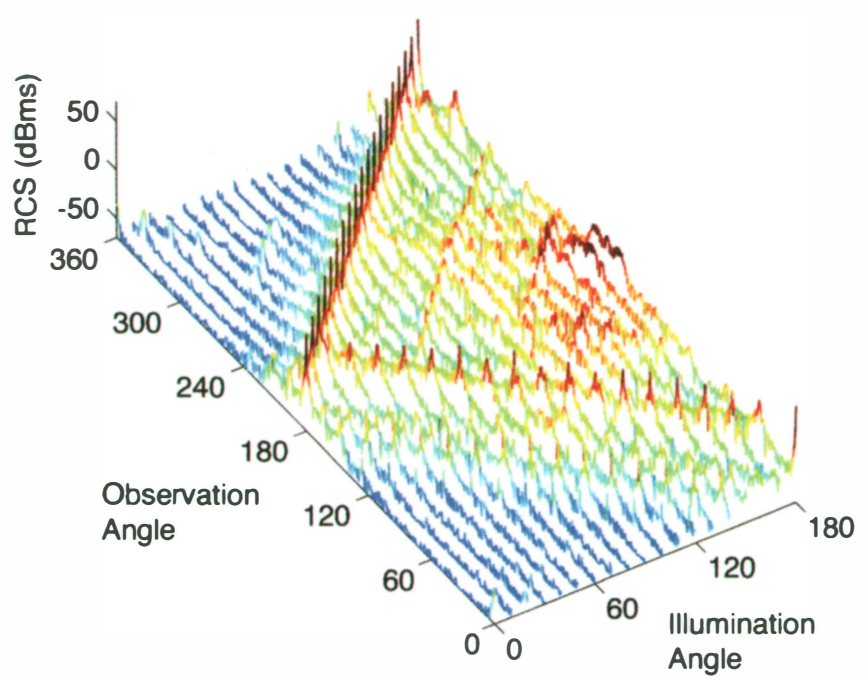

Figure 8. The co-polar bistatic RCS (dBms) of the Flamme in Figure 3d at $360 \mathrm{GHz}$. The RCS was plotted on the $x-y$ plane as a function of the observation and illumination angles when the incident electric field was polarized in (a) the $\phi$ and (b) the $\theta$ directions.

\subsubsection{NASA Almond and the Flamme Discretized with Hundreds of Millions of Unknowns}

Finally, we present the solution of scattering problems involving the NASA Almond and the Flamme discretized with hundreds of millions of unknowns. The NASA Almond was investigated at $1.1 \mathrm{THz}$ and $1.4 \mathrm{THz}$, where its size corresponded to $925 \lambda$ and $1177 \lambda$, and it was discretized with 203,476,224 and $306,696,192$ unknowns, respectively. The Flamme was investigated at $440 \mathrm{GHz}$ and $620 \mathrm{GHz}$, where its size corresponded to $880 \lambda$ and $1240 \lambda$, and it was discretized with $204,664,320$ and 

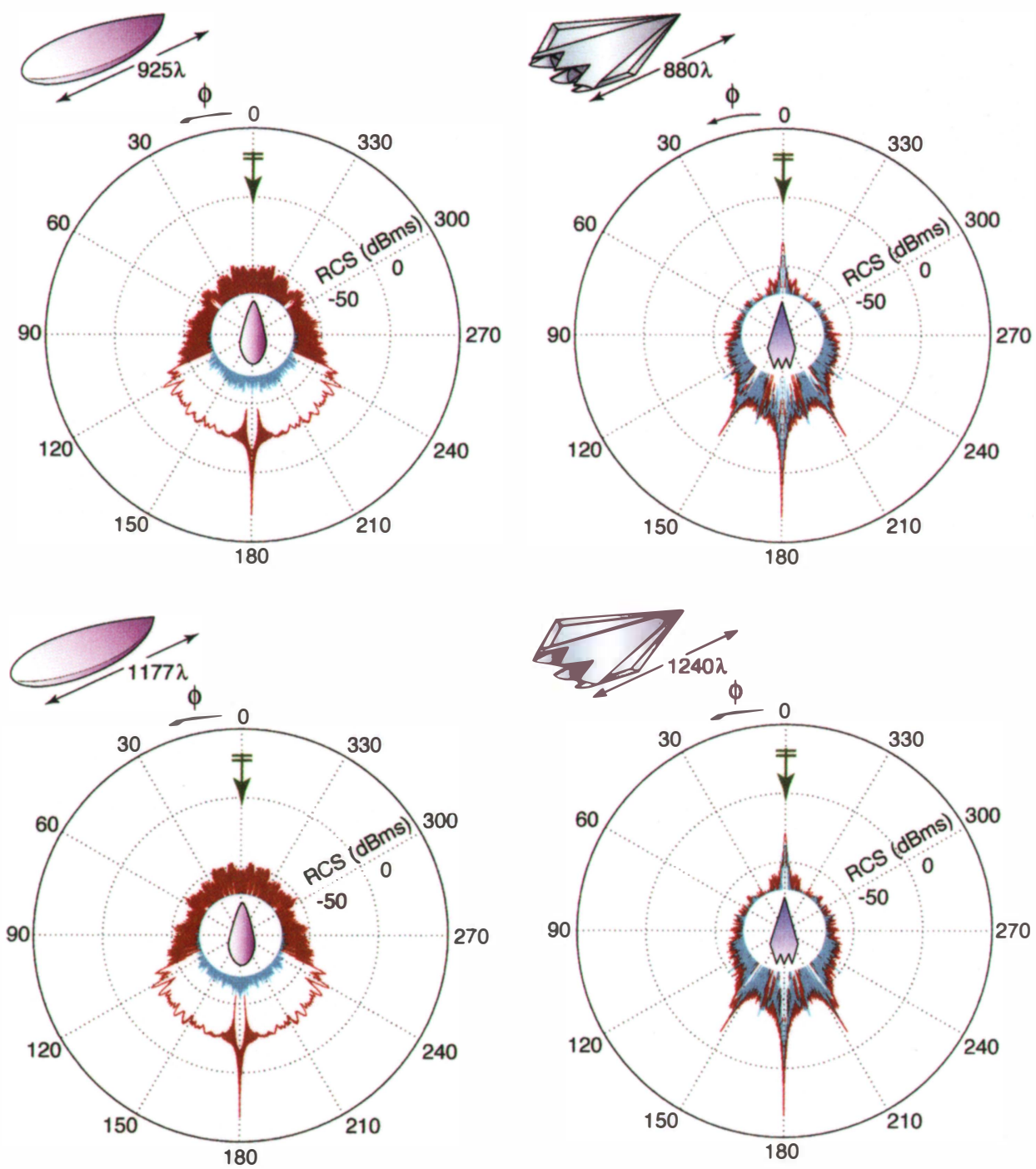

Figure 9. The co-polar (red) and cross-polar (blue) bistatic RCS (dBms) of the NASA Almond in Figure 3c at 1.1 THz and 1.4 THz, and of the Flamme in Figure 3d at $440 \mathrm{GHz}$ and $620 \mathrm{GHz}$. RCS values lower than $-70 \mathrm{dBms}$ have been omitted. Both targets were larger than $1000 \lambda$, and were discretized with more than 300 million unknowns at the higher frequencies. The targets were illuminated by a plane wave propagating in the $-x$ direction, with the electric field polarized in the $\phi$ direction.

$308,289,024$ unknowns, respectively. Both targets were illuminated by a plane wave propagating in the $-x$ direction, with the electric field polarized in the $\phi$ direction. The smaller problems were solved on the Dunnington cluster by employing a 12-level MLFMA. The NASA Almond problem was solved in seven hours, whereas the Flamme problem was solved in 15 hours. The larger problems were solved on the Nehalem cluster by again employing a 12-level MLFMA, and the total processing times were 11 hours and 17 hours for the NASA Almond and the Flamme problems, respectively.

Figure 9 presents the bistatic RCS (dBms) of the NASA Almond and the Flamme on the $x-y$ plane as a function of the bistatic angle $\phi$. We observed that the cross-polar RCS of the NASA Almond was quite low compared to its co-polar RCS at both frequencies. In addition, the co-polar RCS in the back-scattering direction was very low, and the NASA Almond exhibited a stealth ability. For example, at the higher frequency, the backscattered co-polar RCS was $87 \mathrm{~dB}$ lower than the forward-scattered co-polar RCS. It was also remarkable that at both frequencies, there existed no strong specular reflection from the NASA Almond, and its RCS did not exhibit any visible peak, except for in the forward-scattering direction.

As seen in Figure 9, the Flamme also had a stealth ability, with quite low back-scattered RCS compared to the forward-scattered RCS. For example, at the higher frequency, the back-scattered co-polar RCS was $64 \mathrm{~dB}$ lower than the forward-scattered copolar RCS, and the RCS values were extremely low in a wide range of observation angles around the back-scattering direction. On the other hand, as opposed to the RCS of the NASA Almond, the Flamme RCS exhibited two major peaks at around $150^{\circ}$ and $210^{\circ}$, due to the specular reflections from the straight edges and nearly flat surfaces of the target. In addition, the cross-polar RCS of the Flamme was significant and comparable to its co-polar RCS. 


\section{Conclusion}

As reported in this paper, an efficient parallelization of the MLFMA enables rigorous solutions of extremely large electromagnetic problems involving hundreds of millions of unknowns on relatively inexpensive computing platforms with distributed-memory architectures. The effectiveness of the developed simulation environment was demonstrated on various scattering problems involving canonical and complicated objects. Simulations with controllable accuracy provide reliable analysis of electromagnetic problems that cannot be obtained via approximation techniques.

\section{Acknowledgment}

This work was supported by the Scientific and Technical Research Council of Turkey (TUBITAK) under Research Grant 107E136, by the Turkish Academy of Sciences in the framework of the Young Scientist Award Program (LG/TUBA-GEBIP/20021-12), and by contracts from ASELSAN and SSM. Özgür Ergül was also supported by a Research Starter Grant provided by the Faculty of Science at the University of Strathclyde. Computer time was provided in part by generous allocations from Intel Corporation and the Turkish Academic Network and Information Center (ULAKBIM).

The authors would like to thank Jamie Wilcox of Intel Corporation and Onur Temizsoylu of ULAKBIM for the expert technical support and the invaluable assistance they provided to facilitate the experiments on parallel computers.

\section{References}

1. G. Mie, "Contributions to the Optics of Turbid Media, Particularly of Colloidal Metal Solutions," Annals of Physics, 25, 3, 1908, pp. 377-445 (in German).

2. R. Coifman, V. Rokhlin, and S. Wandzura, "The Fast Multipole Method for Wave Equation: A Pedestrian Prescription," IEEE Antennas and Propagation Magazine, 35, 3, June 1993, pp. 7-12.

3. J. Song, C.-C. Lu, and W. C. Chew, "Multilevel Fast Multipole Algorithm for Electromagnetic Scattering by Large Complex Objects," IEEE Transactions on Antennas and Propagation, AP45, 10, October 1997, pp. 1488-1493.

4. W. C. Chew, J.-M. Jin, E. Michielssen, and J. Song, Fast and Efficient Algorithms in Computational Electromagnetics, Norwood, MA, Artech House, 2001.

5. S. Velamparambil, W. C. Chew, and J. Song, "10 Million Unknowns: Is it that Big?," IEEE Antennas and Propagation Magazine, 45, 2, April 2003, pp. 43-58.

6. S. Velamparambil and W. C. Chew, "Analysis and Performance of a Distributed Memory Multilevel Fast Multipole Algorithm," IEEE Transactions on Antennas and Propagation, AP-53, 8, August 2005, pp. 2719-2727.

7. L. Gürel and Ö. Ergül, "Fast and Accurate Solutions of Extremely Large Integral-Equation Formulations Discretised with Tens of Millions of Unknowns," Electronics Letters, 43, 9, April 2007, pp. 499-500.
8. Ö. Ergül and L. Gürel, "Hierarchical Parallelisation Strategy for Multilevel Fast Multipole Algorithm in Computational Electromagnetics," Electronics Letters, 44, 1, January 2008, pp. 3-5.

9. X.-M. Pan and X.-Q. Sheng, "A Sophisticated Parallel MLFMA for Scattering by Extremely Large Targets," IEEE Antennas and Propagation Magazine, AP-50, 3, June 2008, pp. 129-138.

10. Ö. Ergül and L. Gürel, "Efficient Parallelization of the Multilevel Fast Multipole Algorithm for the Solution of Large-Scale Scattering Problems," IEEE Transactions on Antennas and Propagation, AP-56, 8, August 2008, pp. 2335-2345.

11. J. Fostier and F. Olyslager, "An Asynchronous Parallel MLFMA for Scattering at Multiple Dielectric Objects," IEEE Transactions on Antennas and Propagation, AP-56, 8, August 2008, pp. 2346-2355.

12. Ö. Ergül and L. Gürel, "A Hierarchical Partitioning Strategy for an Efficient Parallelization of the Multilevel Fast Multipole Algorithm," IEEE Transactions on Antennas and Propagation, 57, 6, June 2009, pp. 1740-1750.

13. J. M. Taboada, L. Landesa, F. Obelleiro, J. L. Rodriguez, J. M. Bertolo, M. G. Araujo, J. C. Mourino, and A. Gomez, "High Scalability FMM-FFT Electromagnetic Solver for Supercomputer Systems," IEEE Antennas and Propagation Magazine, 51, 6, December 2009, pp. 21-28.

14. A. J. Poggio and E. K. Miller, "Integral Equation Solutions of Three-Dimensional Scattering Problems," in R. Mittra (ed.), Computer Techniques for Electromagnetics, Oxford, Pergamon Press, 1973, Chapter 4.

15. J. R. Mautz and R. F. Harrington, "H-Field, E-Field, and Combined Field Solutions for Conducting Bodies of Revolution," $A E \ddot{U}$, 32, 4, April 1978, pp. 157-164.

16. Ö. Ergül and L. Gürel, "Improving the Accuracy of the Magnetic Field Integral Equation with the Linear-Linear Basis Functions," Radio Science, 41, RS4004, doi:10.1029/2005RS003307, July 2006.

17. M. Benzi, "Preconditioning Techniques for Large Linear Systems: A Survey," Journal of Computational Physics, 182, 2002, pp. 418-477.

18. S. M. Rao, D. R. Wilton, and A. W. Glisson, "Electromagnetic Scattering by Surfaces of Arbitrary Shape," IEEE Transactions on Antennas and Propagation, AP-30, 3, May 1982, pp. 409-418.

19. Ö. Ergül and L. Gürel, "Linear-Linear Basis Functions for MLFMA Solutions of Magnetic-Field and Combined-Field Integral Equations," IEEE Transactions on Antennas and Propagation, AP-55, 4, April 2007, pp. 1103-1110.

20. H. A. van der Vorst, "Bi-CGSTAB: A Fast and Smoothly Converging Variant of Bi-CG for the Solution of Nonsymmetric Linear Systems," SIAM Journal on Scientific and Statistical Computing, 13, 2, March 1992, pp. 631-644.

21. Ö. Ergül and L. Gürel, "Efficient Solution of the Electric-Field Integral Equation Using the Iterative LSQR Algorithm," IEEE Antennas and Wireless Propagation Letters, 7, 2008, pp. 36-39.

22. R. D. Graglia, "On the Numerical Integration of the Linear Shape Functions Times the 3-D Green's Function or its Gradient 
on a Plane Triangle," IEEE Transactions on Antennas and Propagation, AP-41, 10, October 1993, pp. 1448-1455.

23. R. E. Hodges and Y. Rahmat-Samii, "The Evaluation of MFIE Integrals with the Use of Vector Triangle Basis Functions," Microwave and Optical Technology Letters, 14, 1, January 1997, pp. 9-14.

24. P. Ylä-Oijala and M. Taskinen, "Calculation of CFIE Impedance Matrix Elements with RWG and $\hat{n} \times \mathrm{RWG}$ Functions," IEEE Transactions on Antennas and Propagation, AP-51, 8, August 2003, pp. 1837-1846.

25. L. Gürel and Ö. Ergül, "Singularity of the Magnetic-Field Integral Equation and its Extraction," IEEE Antennas and Wireless Propagation Letters, 4, 2005, pp. 229-232.

26. Ö. Ergül, Fast Multipole Method for the Solution of Electromagnetic Scattering Problems, MS Thesis, Bilkent University, Ankara, Turkey, June 2003.

27. S. Koc, J. M. Song, and W. C. Chew, "Error Analysis for the Numerical Evaluation of the Diagonal Forms of the Scalar Spherical Addition Theorem," SIAM Journal on Numerical Analysis, 36, 3, 1999, pp. 906-921.

28. Ö. Ergül and L. Gürel, "Optimal Interpolation of Translation Operator in Multilevel Fast Multipole Algorithm," IEEE Transactions on Antennas and Propagation, AP-54, 12, December 2006, pp. 3822-3826.

29. Ö. Ergül and L. Gürel, "Enhancing the Accuracy of the Interpolations and Anterpolations in MLFMA," IEEE Antennas and Wireless Propagation Letters, 5, 2006, pp. 467-470.

30. A. K. Dominek, M. C. Gilreath, and R. M. Wood, "Almond Test Body," United States Patent 4809003, 1989.

31. L. Gürel, H. Bağcı, J. C. Castelli, A. Cheraly, and F. Tardivel, "Validation through Comparison: Measurement and Calculation of the Bistatic Radar Cross Section (BRCS) of a Stealth Target," Radio Science, 38, 3, RS1046, doi:10.1029/2001RS002583, June 2003. (Aisio)

\section{Changes of Address or Delivery Problems}

Information regarding subscriptions and addresses is managed by IEEE headquarters. It is not maintained, nor can it be changed, by any member of the Magazine staff. If you are a member of the IEEE, your subscription is sent to the address in your IEEE member record. Your address can be confirmed or updated by visiting the Web page dealing with delivery of IEEE publications:

http://www.ieee.org/about/help/subscriptions.html

This page also has information about publication delivery, and a link to an online form that can be used to inquire about missing or delayed publications:

http://www.ieee.org/about/help/publication_inquiry.html

You can also update your address information by contacting IEEE headquarters: Member Address Records, IEEE Headquarters, 445 Hoes Lane, Piscataway NJ 08855-1331 USA; Tel: +1 (908) 981-0060 or +1 (800) 678-4333; Fax: +1 (908) 9819667; E-mail: address.change@ieee.org. If you are an institutional or other non-member subscriber, contact IEEE Customer Service at the above address, telephone, and fax numbers; E-mail: customer.service@ieee.org.

Please do not send requests related to the above items to any member of the Magazine Staff.

\section{Getting the Magazine by Air Freight}

Air freight delivery of the IEEE Antennas and Propagation Magazine is available to subscribers in IEEE Regions 8-10. The cost was recently $\$ 44.00$ per year. This can be added to a member's subscription (and the current cost verified) via the Web at http://www.ieee.org/web/aboutus/help/member_support.html. 